\title{
Beam-based measurement of the center of the new STAR pipe
}

\author{
G. Robert-Demolaize
}

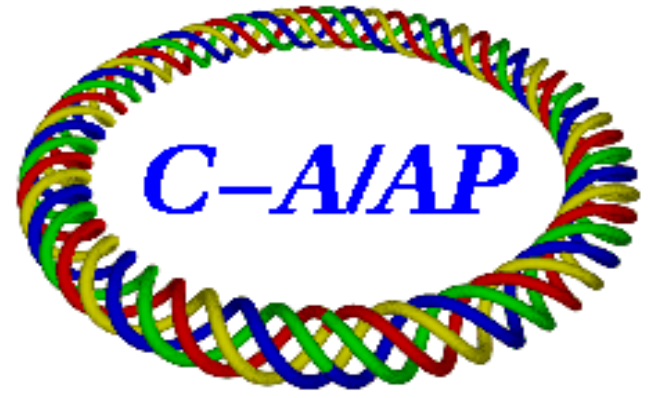

\section{Collider-Accelerator Department Brookhaven National Laboratory Upton, NY 11973}

Notice: This document has been authorized by employees of Brookhaven Science Associates, LLC under Contract No. DE-AC02-98CH10886 with the U.S. Department of Energy. The United States Government retains a nonexclusive, paid-up, irrevocable, world-wide license to publish or reproduce the published form of this document, or allow others to do so, for United States Government purposes. 


\title{
Beam-based Measurement of the Center of the New STAR Pipe*
}

\author{
G. Robert-Demolaize \\ Brookhaven National Laboratory, Upton, NY 11973, U.S.A
}

\begin{abstract}
During the RHIC Shutdown preceding Run13 for polarized protons, various upgrades were brought to the STAR detector, one of which being the partial installation of the Forward GEM Tracker (FGT). This installation includes a new beam pipe at the center of the detector with an internal radius half the size of what the replaced pipe was, from $40 \mathrm{~mm}$ to $20 \mathrm{~mm}$. The following reviews the results of a vertical aperture scans in the STAR interaction region performed at injection energy with both beams, and gives an estimate of the measured transverse offset of the new STAR pipe.
\end{abstract}

\section{INTRODUCTION}

At the beginning of the Run13 Startup, STAR reported seeing high radioactive decay in the detector when trying to take cosmic ray data, an indication that the RHIC beams were irradiating the detector heavily. Since the separation bumps in the lattice design of both beamlines were identical to the ones used in the previous polarized protons run, a misalignment of the new STAR beam pipe with respect the magnets around it could explain the high radiation levels observed. One way to determine whether the pipe is actually off-axis is to scan its vertical aperture with both beams by changing the amplitude of the separation bumps across the interaction region (IR). Since the beam decay signal will increase when the beam reaches the edge of the pipe, the beams will be considered as scraping the pipe when the beam loss rate goes over $50 \%$ per hour. This ensures consistency during the scan for both sides of the aperture and for both beams. Figure 1 shows the amplitude of the vertical separation bumps in STAR that were used for the aperture scan.
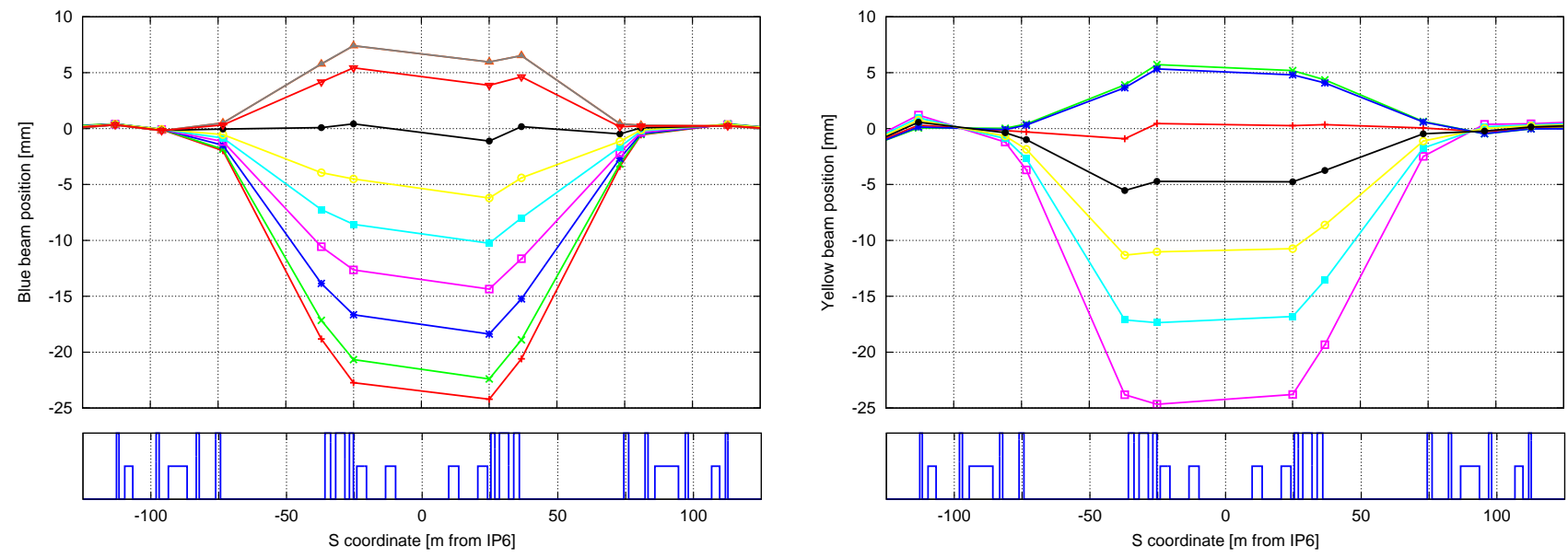

FIG. 1: Top: Amplitudes of the orbit separation bumps in the STAR interaction region (IR6) used for the vertical aperture scan at injection with the Blue beam (left) and the Yellow beam (right). Bottom: Schematic representation of the dipole (long) and quadrupole (tall) magnets along the STAR interaction region for the Blue beam (left) and the Yellow beam (right).

\section{MEASUREMENT}

The aperture scans are performed for both beams at injection energy for the pp13a-v1 ramp with 6 bunches per beam; the corresponding optic functions at the studied beam position monitors (BPM's) are given in Table I, as calculated by the RHIC online model. Figures 2 and 3 show the details of the scans with the Blue and Yellow beam respectively. The normalized $95 \%$ vertical emittance measured by the RHIC Ionization Profile Monitor (IPM) is also shown for both beams. It can be seen of Figure 3 that a large fraction of the beam was lost when scanning the aperture on the way down with the Yellow beam: the beam decay signal remained over 1000\% per hour for a significant amount

${ }^{*}$ The work was performed under the auspices of the US Department of Energy. 
TABLE I: Optic functions at injection energy for the pp13a-v1 ramp and locations of the BPM's used for the STAR pipe aperture scan. The longitudinal position $s$ is given at the end of each BPM with respect to the interaction point IP6 in the STAR interaction region in the clockwise direction. The optic functions at the DX BPM's are given for both the (B)lue and (Y)ellow beam.

\begin{tabular}{|l|cc|cc|cc|}
\hline BPM name & g5-bx & g6-bx & bi5-b1 & bo6-b1 & yo5-b1 & yi6-b1 \\
\hline S [m] from IP6 & -8.326 & 7.993 & -25.024 & 25.024 & -25.024 & 25.024 \\
\hline$\beta_{X}[\mathrm{~m}]$ & $26.010(\mathrm{~B})$ & $12.387(\mathrm{~B})$ & 88.452 & 48.164 & 47.185 & 82.733 \\
& $13.524(\mathrm{Y})$ & $24.676(\mathrm{Y})$ & & & & \\
\hline$\beta_{y}[\mathrm{~m}]$ & $15.969(\mathrm{~B})$ & $16.698(\mathrm{~B})$ & 80.375 & 84.578 & 83.531 & 77.658 \\
& $17.678(\mathrm{Y})$ & $15.134(\mathrm{Y})$ & & & & \\
\hline
\end{tabular}
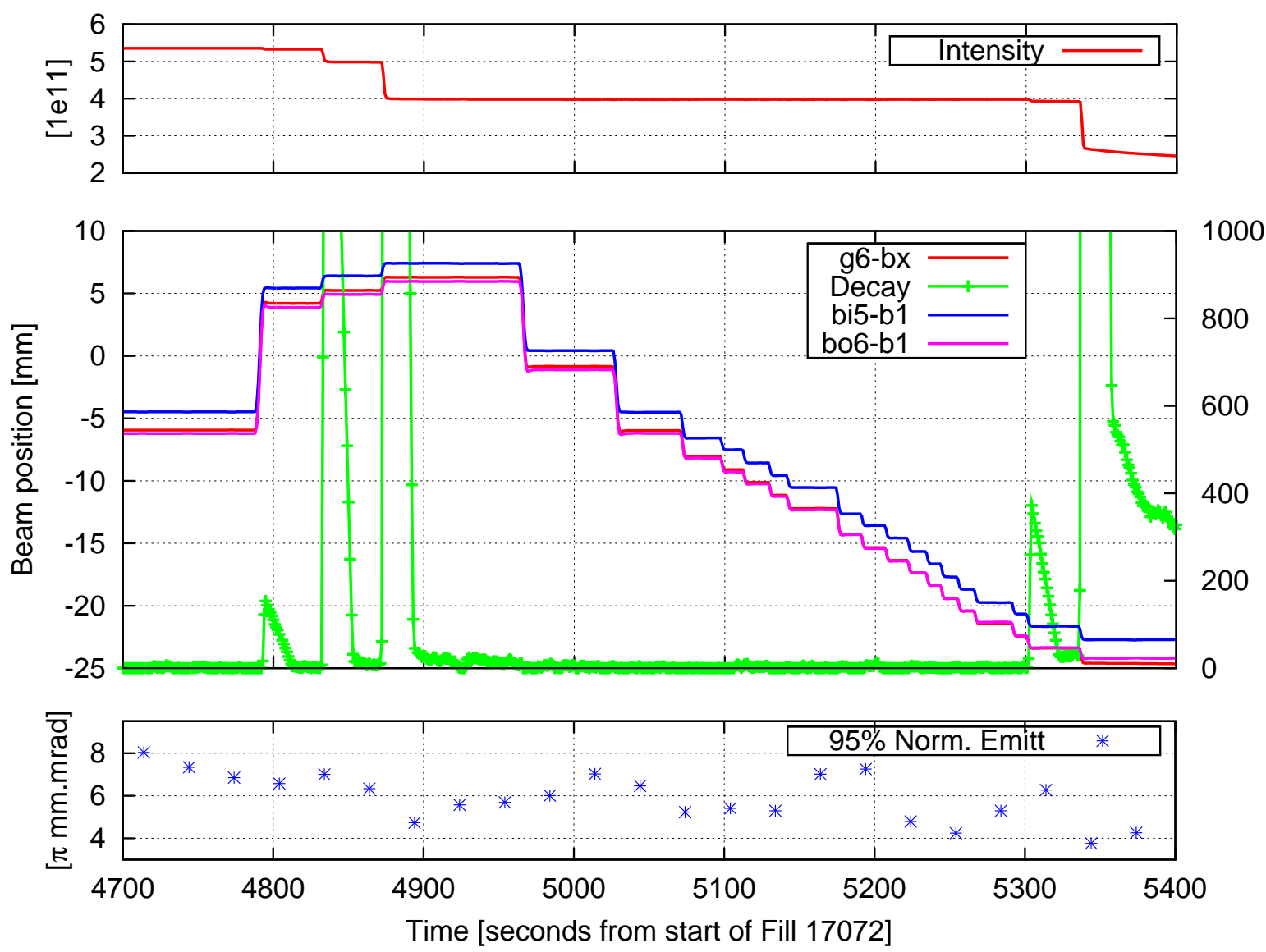

FIG. 2: Top: Blue proton beam intensity. Center: Blue beam decay rate and vertical position at the DX and Q1 BPM's as a function of time during Fill \#17072 for the aperture scan in STAR. Only the g5-bx BPM reported data for the Blue beam during that scan due to a timing setup problem. Bottom: Normalized vertical emittance as measured by the RHIC IPM.

of time after the last step, and the normalized emittance dropped from $8 \pi$ to $6 \pi$. To get an accurate measurement of the STAR pipe offset, one has calculate the distance between its two edges by considering both the position of the center of the beam (given by the amplitude of the separation bump) and the beam size as measured by the IPM.

Table II compiles the result at the DX BPM's (closest to the new STAR pipe) for both aperture scans. Since this measurement was performed early in the Run13 startup period, some of the BPM's did not have their timing properly set up: that was the case for g5-bx for the Blue beam, which was reporting data for the Yellow beam. Therefore the only DX BPM used for the aperture scan in Blue is g6-bx. 

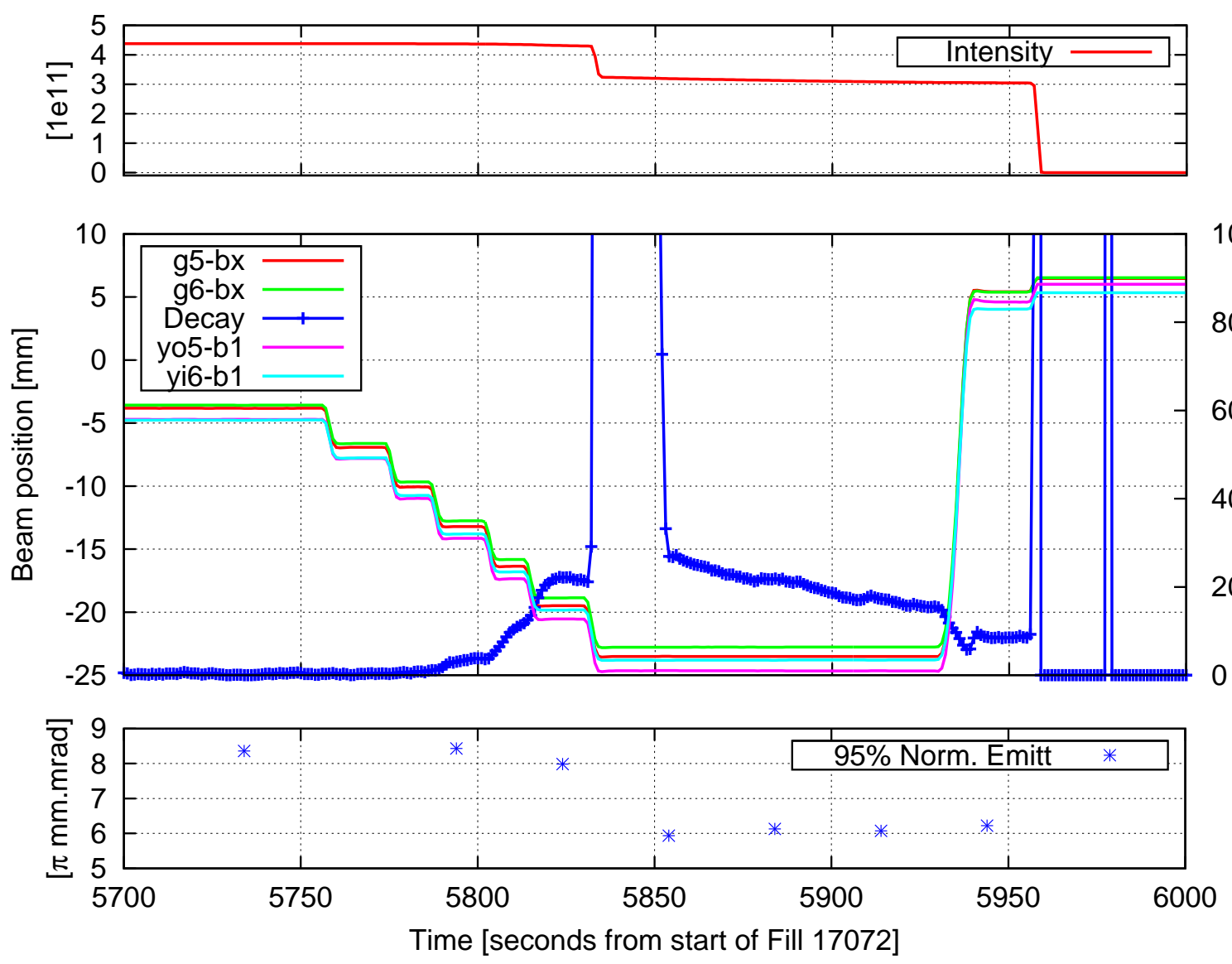

FIG. 3: Top: Yellow proton beam intensity. Center: Yellow beam decay rate and vertical position at the DX and Q1 BPM's as a function of time during Fill \#17072 for the aperture scan in STAR. Bottom: Normalized vertical emittance as measured by the RHIC IPM.

TABLE II: Overview of the STAR aperture scan with the Blue (top) and Yellow (bottom) beams during Fill \#17072. The beam positions reported for each BPM are derived from the separation bump amplitude that causes the beam decay rate to go over the threshold of $50 \%$ previously established. The position of the aperture limit and center of the new STAR pipe are interpolated from the BPM readings, the measured beam emittance and the optic functions from Table I. All positions are given with the expected center of the STAR pipe as the "zero" reference position.

\begin{tabular}{|l|c|c|c|c|c|}
\hline Beam / Scan & $\begin{array}{c}\text { Position at g5-bx } \\
{[\mu \mathrm{m}]}\end{array}$ & $\begin{array}{c}\text { Position at g6-bx } \\
{[\mu \mathrm{m}]}\end{array}$ & $\begin{array}{c}95 \% \text { Vertical normalized emittance } \\
{[\pi \text { mm.mrad }]}\end{array}$ & $\begin{array}{c}\text { Aperture limit } \\
{[\mu \mathrm{m}]}\end{array}$ & $\begin{array}{c}\text { Center of the pipe } \\
{[\mu \mathrm{m}]}\end{array}$ \\
\hline Blue / Up & 4009.12 & N/A & 6.6738 & 5056.87 & -9511.811 \\
Blue / Down & -23096.8 & N/A & 5.8827 & -24080.49 & 3536.47 \\
\hline Yellow / Up & 2498 & 2526 & 6.1929 & -6857.638 \\
Yellow / Down & -16354.08 & -15788.42 & 8.2769 & -17554.63 & \\
\hline
\end{tabular}

\section{CONCLUSION}

From the results of calculations shown in Table II, the measured offset of the new STAR pipe in the vertical plane is about $-9.5 \mathrm{~mm}$ in Blue and $-6.9 \mathrm{~mm}$ in Yellow. The discrepancy of $2.6 \mathrm{~mm}$ between the two could be attributed in part to the resolution of the measurements, in particular of the normalized emittance of the Blue beam as evidenced by the fluctuations seen in Figure 2. Considering that the beam offset used for the entire Run13 operations was set at $-5 \mathrm{~mm}$ (setting the separation bumps in the STAR interaction region at $+0 \mathrm{~mm}$ for Blue and $-10 \mathrm{~mm}$ for Yellow), the conservative approach pushes for a recommendation to raise the new STAR pipe by $6.9 \mathrm{~mm}$ in the vertical plane. 